\title{
Size-controlled fabrication of zein nano/ microparticles by modified anti-solvent precipitation with/without sodium caseinate
}

This article was published in the following Dove Press journal:

International Journal of Nanomedicine

10 November 2017

Number of times this article has been viewed

Feng Li'
Yan Chen',2
Shubo Liu'
Jian Qi'
Weiying Wang'
Chenhua Wang'
Ruiyue Zhong'
Zhijun Chen'
Xiaoming Li'
Yuanzhou Guan'
Wei Kong',2
Yong Zhang',2
'National Engineering Laboratory for
AlDS Vaccine, School of Life Sciences,
jilin University, Changchun, ${ }^{2}$ Key
Laboratory for Molecular Enzymology
and Engineering, the Ministry of
Education, School of Life Sciences,
Jilin University, Changchun, China

Jilin University, Changchun, China

\begin{abstract}
Zein-based nano/microparticles have been demonstrated to be promising carrier systems for both the food industry and biomedical applications. However, the fabrication of size-controlled zein particles has been a challenging issue. In this study, a modified anti-solvent precipitation method was developed, and the effects of various factors, such as mixing method, solvent/anti-solvent ratio, temperature, zein concentrations and the presence of sodium caseinate (SC) on properties of zein particles were investigated. Evidence is presented that, among the previously mentioned factors, the mixing method, especially mixing rate, could be used as an effective parameter to control the size of zein particles without changing other parameters. Moreover, through fine-tuning the mixing rate together with zein concentration, particles with sizes ranging from nanometers to micrometers and low polydispersity index values could be easily obtained. Based on the size-controlled fabrication method, SC-coated zein nanoparticles could also be obtained in a size-controlled manner by incubation of the coating material with the already-formed zein particles. The resultant nanoparticles showed better performance in both drug loading and controlled release, compared with zein/SC hybrid nanoparticles fabricated by adding aqueous ethanol solution to SC solution. The possible mechanisms of the nanoprecipitation process and self-assembly formation of these nanoparticles are discussed.
\end{abstract}

Keywords: zein, phase separation, microparticle, nanoparticle, encapsulation, drug release

\section{Introduction}

Plant proteins are renewable and biodegradable, and some of them (eg, zein, soybean, and wheat proteins) have been used as various functional materials in food and drug industries. ${ }^{1}$ Among these, zein and zein-based carriers have drawn much attention from experts in both food and pharmaceutical fields due to its molecular characterization, cytocompatibility, and biodegradability. ${ }^{2-5}$ Zein belongs to the class of prolamin proteins and is the main storage protein of maize. Zein is primarily present as protein bodies in endosperm cells and can be extracted and separated with various technologies. ${ }^{6,7}$ According to solubility and sequence similarity, zein can be further divided into $\alpha$-, $\beta$-, $\gamma$-, and $\delta$-zeins. Among these, $\alpha$-zein accounts for the largest fraction (approximately $80 \%$ ) of the total zein mass. Commercial zein usually refers to $\alpha$-zein with a purity higher than $90 \%$. Numerous studies have demonstrated that zein could be fabricated into various carriers, such as films, ${ }^{3}$ nanofibers, ${ }^{4}$ nano/microparticles, ${ }^{8}$ nano/microcapsules, ${ }^{9,10}$ and micelles. ${ }^{11}$ These carriers and their biomedical applications have been reviewed recently. ${ }^{3-5}$

Currently, although zein film coating for tablets and pellets is the only approved biomedical usage by the US Food and Drug Administration (FDA), other zein-based
Correspondence: Yong Zhang

School of Life Sciences, Jilin

University, 2699 Qianjin Street,

Changchun 1300 I2, China

$\mathrm{Tel}+8643$ I 85167751

Fax +8643185167674

Email zhangyongkingl@gmail.com
International Journal of Nanomedicine 2017:12 8|97-8209

8197

Dovepress f in 0

http://dx.doi.org/10.2147/1/N.S143733 
carriers (eg, nano/microparticles) have shown great potential in both food and biomedical applications. ${ }^{2-5}$ The fabrication of zein-based nano/microparticles can be realized with various methods, including chemical crosslinking, ${ }^{12}$ antisolvent precipitation, ${ }^{13}$ supercritical anti-solvent technique, ${ }^{14}$ spray drying, ${ }^{15,16}$ emulsification-solvent evaporation, ${ }^{17}$ and emulsification-precipitation-gelation technique. ${ }^{18}$ These methods involve different mechanisms and vary in their simplicity and scale-up potential. Related information is discussed in our recently published review. ${ }^{5}$

Among the previously mentioned methods, anti-solvent precipitation, also known as nanoprecipitation or phase separation, has drawn increased interest due to its simplicity and scalable potential. The anti-solvent precipitation method is proposed to be involved in three processes: clustering, nucleation, and nucleus growth. ${ }^{19,20}$ These processes essentially shift the solute state during the change in solvent composition. Thus, solute concentration, mixing method, solvent/anti-solvent ratio and compositions, and the absence/ presence of additives may exert various effects on the previously mentioned processes and therefore the properties of resultant particles, such as size, size distribution, and surface characteristics. For example, low zein concentration, increased shear rate or high initial alcohol concentration have been reported to lead to a smaller particle size. ${ }^{13,21}$ In the case of using an alcohol aqueous solution as the solvent and water as the anti-solvent, the sizes of the resultant zein nanoparticles produced ranked in order with the following solvents: ethanol $>$ isopropanol $>$ methanol. ${ }^{22}$ Sodium caseinate (SC) and chitosan remarkably improved the stability of zein nanoparticles due to the supportive surface charge characteristics. ${ }^{23,24}$ These modified particle properties can be used as an efficient strategy to obtain the desired delivery systems in terms of drug encapsulation, release, and biomedical applications. For instance, controlled and delayed release of gitoxin could be achieved by encapsulation into zein microparticles fabricated with ethanol aqueous solution, while those prepared with methanol or isopropanol aqueous solutions showed faster release profiles. ${ }^{25}$ Zein nanoparticles with improved cellular uptake and transport efficiency across a Caco- 2 cell monolayer could be obtained by introduction of $\mathrm{SC}$ into the formulation. ${ }^{26}$ Citric acid-cross-linked zein nanocapsules showed kidney-targeted delivery and distribution, while uncross-linked ones mainly accumulated in the liver and spleen in mice after intravenous administration. ${ }^{9}$

In this study, a modified anti-solvent precipitation method, without using special devices, (eg, microfluidic devices or chamber mixers) was developed. The aim is to achieve size-controlled fabrication of zein nano/microparticles while keeping a narrow size distribution. Particle size is a key parameter for drug and nutrition delivery, as the size can significantly influence the interactions of particles with cells and therefore their absorption and distribution in vivo. ${ }^{27}$ Moreover, key factors and related mechanisms were also investigated and discussed. As far as we know, this study is the first report on size-controlled fabrication of zein particles through adjusting a single factor. In addition, SC-coated and size-controlled zein nanoparticles were also developed and compared with $\mathrm{SC} /$ zein hybrid nanoparticles in terms of particle properties, drug encapsulation and release profiles.

\section{Materials and methods Materials}

Zein from maize, SC from bovine milk, and coumarin 6 with purity of $98 \%$ were purchased from Sigma-Aldrich Co. (St Louis, MO, USA). Deionized water was used throughout the study. All other chemicals were of analytical reagent grade or purer.

\section{Preparation of zein nanoparticles}

Blank zein nanoparticles were fabricated with an anti-solvent precipitation method. Briefly, zein powder was dissolved in $5 \mathrm{~mL} 80 \%$ ethanol (ethanol/water, 80:20, v/v), followed by the addition of $15 \mathrm{~mL}$ deionized water with continuous stirring. The speed of the addition of deionized water was controlled by a peristaltic pump BT300-1F (Longer Precision Pump Corporation, Baoding, China). The stirring continued at 1,200 rpm for $3 \mathrm{~h}$ to ensure that the ethanol was completely evaporated. Moreover, effects of mixing-sequence, needle position, temperature, ethanol/water ratio, zein concentration, and rate of solvent addition on the properties of particles were investigated to illustrate the key factors. In order to preserve their inherent properties, the resultant particles were directly used for physicochemical characterization without centrifugation and re-dispersion treatment. These processes were demonstrated to cause smaller particle sizes and broader size distribution and thus higher heterogeneity of nanoparticles. Moreover, some particles could not be suspended and strongly adhered onto the bottom of tube after centrifugation, suggesting a decreased efficiency in the production of particles.

\section{Preparation of zein/SC hybrid nanoparticles and SC-coated zein nanoparticles}

Zein/SC hybrid nanoparticles were prepared using a similar method to that previously mentioned. Briefly, $50 \mathrm{mg}$ zein and 
$50 \mathrm{mg}$ SC were dissolved in $5 \mathrm{~mL} \mathrm{80 \%} \mathrm{ethanol} \mathrm{and} 15 \mathrm{~mL}$ deionized water, respectively. The aqueous alcohol solution of zein was then added to the solution containing SC while stirring at 1,200 rpm for $3 \mathrm{~h}$. The effect of mixing speed on the properties of particles was also evaluated. Moreover, $\mathrm{SC}$-coated zein nanoparticles were fabricated with the following two steps. First, zein nanoparticles were prepared using a similar anti-solvent precipitation method as that for blank zein nanoparticles but with a shorter ethanol evaporation time of $0.5 \mathrm{~h}$. Second, the resultant zein nanoparticle suspension was directly poured into a $15 \mathrm{~mL}$ SC solution $(3.33 \mathrm{mg} / \mathrm{mL})$ while stirring at 1,200 rpm for $2.5 \mathrm{~h}$.

For drug encapsulation, coumarin $6(0.2 \mathrm{mg} / \mathrm{mL})$ was directly dissolved in $5 \mathrm{~mL} 80 \%$ ethanol solution with zein. The fabrication process was the same as that for zein/SC hybrid nanoparticles and SC-coated zein nanoparticles. The resultant drug-loaded nanoparticles were centrifuged for further analysis of encapsulation efficiency. For the drug release study, the re-dispersed nanoparticle suspension was directly used without further lyophilization and re-hydration treatment.

\section{Transmission electron microscopy (TEM)}

The morphology of the previously mentioned nanoparticles was observed using a transmission electron microscope (Tecnai G² F20 S-Twin, Hillsboro, OR, USA). The nanoparticle suspension was diluted with deionized water and cast-dried onto a 150-mesh carbon-coated copper grid. The images were taken at the appropriate magnifications.

\section{Fourier transform infrared spectroscopy (FTIR)}

FTIR spectra measurements were performed using Bruker Vertex $80 \mathrm{~V}$ instrument equipped with a DTGS detector (Bruker Corporation, Billerica, MA, USA). Data were collected on the transmittance mode over a frequency region of $4,000-400 \mathrm{~cm}^{-1}$ for 32 interferograms with a resolution of $4 \mathrm{~cm}^{-1}$ at $25^{\circ} \mathrm{C}$. Samples were prepared by mixing $150 \mathrm{mg}$ $\mathrm{KBr}$ with $1 \mathrm{mg}$ sample followed by compressing processes. Finally, the spectra were presented in absorption mode after the baseline correction.

\section{Particle size, polydispersity index (PDI), and zeta potential}

The sizes and PDI of nanoparticles were determined by Zetasizer Nano ZS (Malvern Instruments, Malvern, UK) following the principles of dynamic light scattering. Samples were diluted at appropriate rates, and the temperature was maintained at $25^{\circ} \mathrm{C}$. In addition, zeta potentials were determined based on the electrophoretic mobility of the nanoparticles in $1 \mathrm{mM}$ sodium chloride solution with the same instrument at $25^{\circ} \mathrm{C}$.

\section{Encapsulation efficiency}

After the particle preparation, a certain volume of the suspension solution was sampled and centrifuged at $8,000 \times g$ for 15 min. The drug content of the supernatant was measured using a fluorospectrometer for coumarin 6. The intrinsic fluorescence of coumarin 6 was measured using $450 \mathrm{~nm}$ excitation and the emission was monitored in the range of 470-650 nm. The excitation and emission slits were 5 and $5 \mathrm{~nm}$, respectively. All background effects were subtracted. The encapsulation efficiency of the drug was calculated by subtracting the unloaded amount of drug by the initial amount of drug, and then followed by dividing by the initial amount of drug. The results are presented as an average of the triplicate measurements.

\section{Drug release}

The drug release profile of the nanoparticles was determined with the positive dynamic method. Briefly, drug-loaded nanoparticles in $1 \mathrm{~mL}$ were dispersed in $29 \mathrm{~mL}$ phosphate buffered solution containing $0.5 \%$ Tween 20, pH 7.4 (PBST) ${ }^{28}$ Here, Tween 20 was used to solubilize the released coumarin 6 and to create sink condition. Under the linear shaking bath conditions $\left(37^{\circ} \mathrm{C}, 100 \mathrm{rpm}\right)$, an appropriate volume of suspension was taken at the set time for the detection of the drug content as previously described for measurement of the encapsulation efficiency. Meanwhile, the same volume of PBST was added until the drug release reached the highest value.

\section{Results and discussion Effect of mixing methods and ethanol/ water ratio}

\section{Mixing-sequence}

In this study, an anti-solvent precipitation method was used to fabricate zein nanoparticles. Deionized water was employed as the anti-solvent, while an aqueous ethanol solution was used as the solvent for zein. To illustrate the possible effect of mixing-sequence on properties of zein nanoparticles, adding the solvent to anti-solvent was compared with adding the anti-solvent to solvent at the mixing rate of $30 \mathrm{~mL} / \mathrm{min}$. In both cases, the amounts of zein, solvent, and anti-solvent were kept the same. As shown in Figure 1, following the addition of aqueous ethanol solution into deionized water, the resultant particles showed a broad size distribution of approximately $110 \mathrm{~nm}$ accompanied by the appearance of 


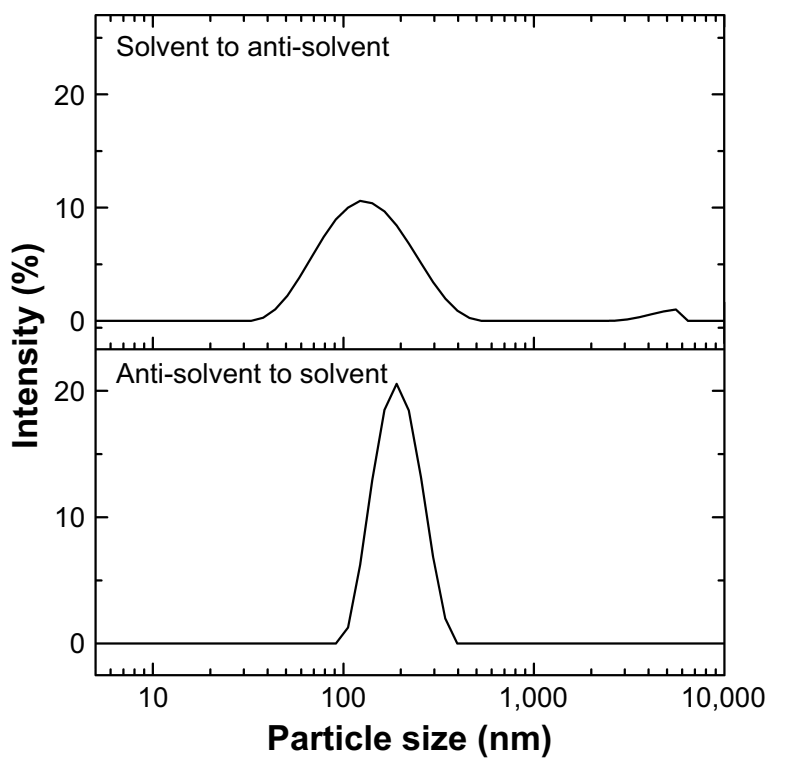

Figure I Effect of mixing sequence on the particle size and size distribution of zein nanoparticles.

micrometer-scale zein aggregates. The PDI of this system was 0.34 , indicating a high polydispersity. In contrast, introduction of anti-solvent into the aqueous ethanol solution gave a narrower size distribution of approximately $187 \mathrm{~nm}$ and a PDI value lower than 0.06, indicating nearly monodispersion. The previously mentioned results indicate that the mixing-sequence can exert a significant influence on the final state of the resultant nanoparticles.

The variant sizes and size distributions may be explained by the different nanoprecipitation processes occurring during mixing. Continuously adding zein-containing solvent into anti-solvent can lead to recurrent and transient supersaturation of zein due to rapid ethanol diffusion and therefore repeated nucleation. With further addition of zein-containing aqueous ethanol solution, dissolved zein will be consumed competitively by nucleation and nuclear growth over a short time. Early formed nuclei have a greater chance of growing into large particles via nucleus collision and zein molecule absorption, while late-generated nuclei would have less of such an opportunity and ultimately experience limited growth. As a result, a broad size distribution and even large aggregates would occur in this system. In the case of adding anti-solvent into zein-containing aqueous ethanol solution, zein super-saturation can only happen when the amount of anti-solvent reaches up to a certain volume. Thus, transient super-saturation at a high zein concentration would occur and produce massive nuclei. These nuclei will have the same opportunity for growth following the further addition of anti-solvent. As a result, a similar particle size and low size distribution can be obtained. The previously mentioned speculation agrees well with the established "burst nucleation" concept in the synthesis of monodisperse nanocrystals and nanoparticles. ${ }^{29,30}$ In these reports, renucleation or concurrent nucleation with particle growth were attempted to avoid obtaining polydisperse particles. In the subsequent experiments, adding deionized water into zein-containing aqueous ethanol solution was used to obtain zein nanoparticles of high quality unless otherwise specified.

\section{Needle position}

The effect of needle position on properties of zein nanoparticles was also investigated. The results are shown in Figure 2. Unexpectedly, the needle position showed a remarkable influence on both the particle size and size distribution. Keeping the needle above the surface of the zein-containing aqueous ethanol solution led to smaller particle sizes and lower PDI values, although all cases showed a nearly mono-dispersed state of zein nanoparticles. Interestingly, with the increasing mixing rate, the effect of needle position on particle properties was undermined. For example, when the addition rate of the water phase remained at $60 \mathrm{~mL} / \mathrm{min}$, particles prepared by keeping the needle above and below the liquid surface displayed the same size of approximately $173 \mathrm{~nm}$ and narrower gap between their PDI values. However, with decreasing addition rates of the water phase, increasing particle sizes and larger gaps between PDI values were observed in corresponding groups with different needle positions. These results suggested that the mixing rate had an overwhelming effect on particle properties in comparison with the needle position.

The effect of needle position can be explained by the variant diffusion and mixing efficiency under the different

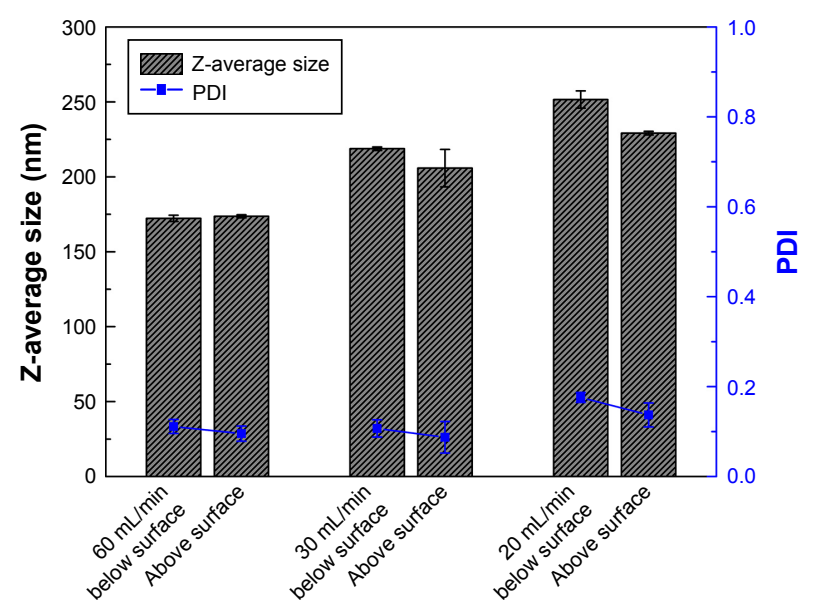

Figure 2 Effect of needle position on the particle size and size distribution of zein nanoparticles.

Abbreviation: PDI, polydispersity index. 
conditions. As ethanol is less dense than water, when the needle was placed under the surface of the zein-containing aqueous ethanol solution, the added water tended to remain at the bottom of the vessel. This situation resulted in a relatively lower mixing efficiency compared with the case of putting the needle above the solution surface. In addition, in the latter case, the collision of added water flow with zein-containing ethanol solution resulted in rapid diffusion and mixing. The high mixing efficiency means a shorter time to complete mixing and therefore a lower chance of renucleation. As mentioned previously, less renucleation would result in improved monodispersity. The previously mentioned speculation was further supported by the overwhelming effect of increased mixing rate on particle size and PDI values. Considering the requirement for producing high quality particles, the needle was kept above the solution surface in the subsequent experiments.

\section{Ethanol/water ratio}

The influence of ethanol/water ratios on particle properties was also studied. In this experiment, the mixing rate of $30 \mathrm{~mL} / \mathrm{min}$ was chosen as a representative one to obtain zein nanoparticles of high quality. The aqueous ethanol solution (50 mg zein, 80\% ethanol, v/v) was also kept constant at $5 \mathrm{~mL}$, but the volume of anti-solvent (ie, deionized water) was varied from $15 \mathrm{~mL}$ to $5 \mathrm{~mL}$ at $2.5 \mathrm{~mL}$ increments. This design ensured the same composition at the starting point in the ternary phase diagram, while the ending points after mixing fell into different areas in the ternary phase diagram of zein in ethanol and water. That is, the ending points of formulations 1-3 were in precipitation area, that of formulation 4 was near the borderline of precipitation and coacervation, and that of formulation 5 fell into coacervation area. The phase areas mentioned here are based on the work published by Mosse. ${ }^{31}$ As shown in Table 1, with the decrease in volumes of anti-solvent from $15 \mathrm{~mL}$ to $7.5 \mathrm{~mL}$, particle sizes increased from $187.1 \mathrm{~nm}$ to $208.7 \mathrm{~nm}$. In addition, all mixtures produced mono-dispersion systems as evidenced by the small PDI values lower than 0.08 . However, a large particle size and high PDI were observed in the case with $5 \mathrm{~mL}$ anti-solvent, indicating poor particle quality.
Although both coacervation and precipitation can produce remarkable turbidity, they are intrinsically different phenomena. The former is reversible and refers to liquid-liquid separation, whereas the latter is irreversible and involves liquid-solid separation. ${ }^{32}$ Coacervation usually occurs just before precipitation. ${ }^{33}$ Precipitation requires tight intermolecular interactions and will cause extensive expulsion of water molecules and counter ions, while coacervation does not. Falling into the coacervation area means that nucleation and particle growth of zein will be delayed and happen over a long time with ethanol evaporation, while these processes will occur more rapidly when directly shifting the mixed system toward precipitation by adding more anti-solvent. In a previous study, nanoparticle formation at a high anti-solvent/solvent ratio was described via the nucleation-aggregation mechanism, while it mainly followed the nucleation-and-growth mechanism at a low anti-solvent/solvent ratio. ${ }^{19}$ However, according to the burst nucleation concept discussed previously, it is also predicable that high ethanol/water ratios will result in large particles and high PDI values. Considering the high particle quality, $15 \mathrm{~mL}$ of anti-solvent was used in the subsequent experiments, ie, the endpoint of the mixing system was kept in the precipitation range.

\section{Size-controlled preparation of zein nano/microparticles Mixing rate}

Inspired by the findings on the effect of needle position, the influence of mixing rate on properties of zein nanoparticles was investigated in detail. As shown in Figure 3A, at the highest mixing rate of $60 \mathrm{~mL} / \mathrm{min}$, the resultant particles showed a size of $165 \mathrm{~nm}$ and a small PDI value of 0.087 . However, with the decrease in rate of addition of anti-solvent into solvent, increasing particle sizes and slightly higher PDI values were observed. At the mixing rate of $3.87 \mathrm{~mL} / \mathrm{min}$, large particles with a size of $470 \mathrm{~nm}$ were obtained, accompanied with a higher PDI value of 0.20 . The results indicated a significant influence of mixing rate on the size and size distribution of zein nanoparticles. The tendency of nanoparticle

Table I The influence of ethanol/water ratios on particle properties

\begin{tabular}{|c|c|c|c|c|c|}
\hline Aqueous ethanol solution:water (v/v) & $4: 6$ & $4: 8.5$ & $4: 11$ & $4: 13.5$ & $4: 16$ \\
\hline Z-average size $(\mathrm{nm})$ & $403.0 \pm 2.89$ & $208.7 \pm 3.25$ & $200.2 \pm 5.72$ & $201.2 \pm 4.19$ & $187.1 \pm 0.90$ \\
\hline 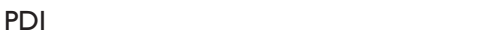 & $0.280 \pm 0.01$ & $0.072 \pm 0.01$ & $0.05 \pm 0.02$ & $0.066 \pm 0.02$ & $0.063 \pm 0.02$ \\
\hline Zeta potential (mV) & $40.1 \pm 0.82$ & $39.7 \pm 1.10$ & $35.7 \pm 0.59$ & $36.2 \pm 2.35$ & $35.63 \pm 0.59$ \\
\hline Formulation number & 5 & 4 & 3 & 2 & I \\
\hline
\end{tabular}

Note: Data presented as mean \pm standard deviation.

Abbreviation: PDI, polydispersity index. 


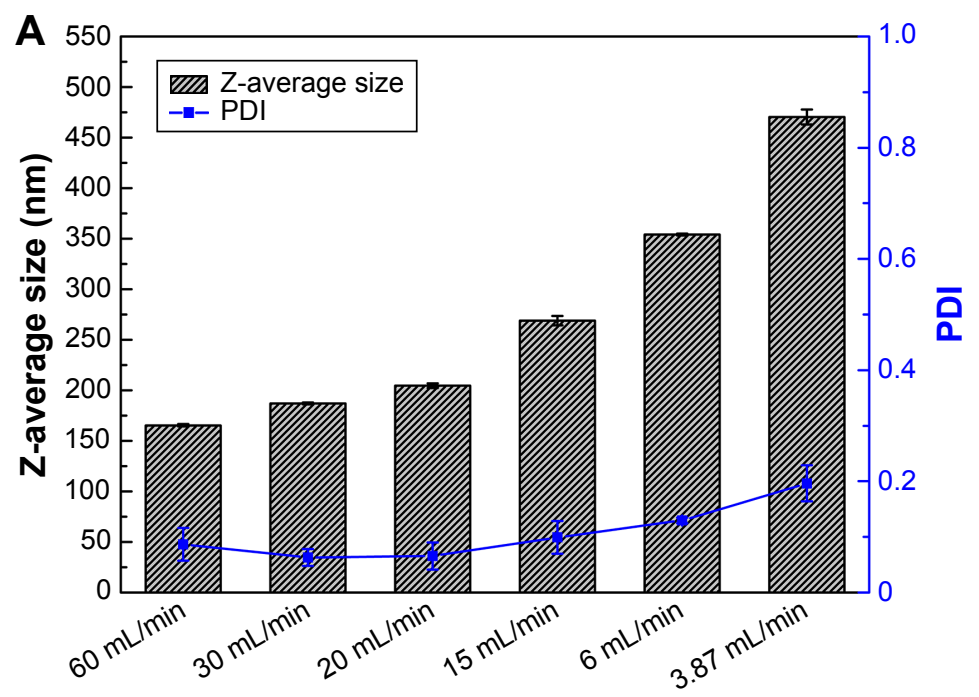

\section{Adding rate}

B
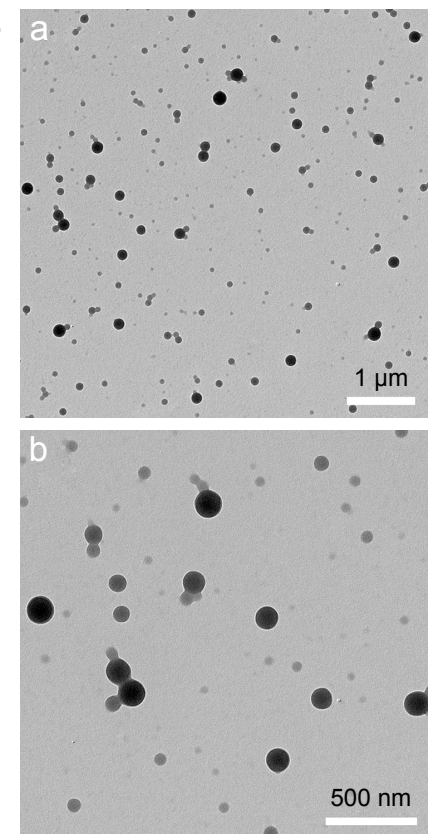
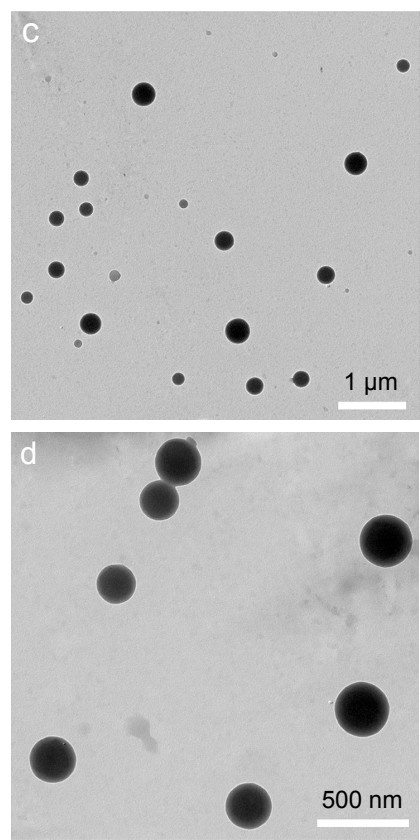
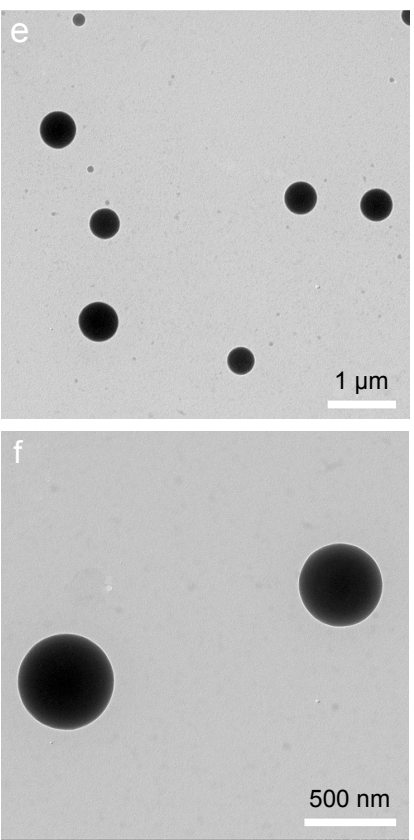

Figure 3 (A) Effect of mixing rate on particle size and size distribution of zein nanoparticles. (B) TEM images of zein nanoparticles fabricated with different mixing rates of $60 \mathrm{~mL} / \mathrm{min}$ (a and b), $15 \mathrm{~mL} / \mathrm{min}$ (c and d) and $3.87 \mathrm{~mL} / \mathrm{min}$ (e and f).

Abbreviations: PDI, polydispersity index; TEM, transmission electron microscopy.

sizes to change with mixing rate remained even after the particles were subjected to centrifugation and redispersion treatment. Further morphological analysis was performed on the resultant zein particles, and the results are shown in Figure 3B. All particles fabricated in different mixing rates had a spherical shape. However, the particle size increased along with the decreased mixing rate, which agreed well with the previously mentioned dynamic light scattering data. The previously mentioned findings indicated that size-controlled zein particles with low PDI could be easily fabricated by only adjusting the addition rate of the anti-solvent.
Size-controlled fabrication of particles usually requires special devices, such as well-designed microfluidic devices or chamber mixers. ${ }^{34-36}$ These special devices can achieve a kinetically controlled nanoprecipitation and therefore well-controlled particle size via a geometrical confinementbased mixing process. Here, we demonstrated that the controlled nanoprecipitation could also be achieved in an optimized mixing system through adjusting a single parameter. The effect of mixing rate on particle sizes can be explained by a fundamental equation for size control of monodisperse systems. ${ }^{29}$ The final particle number of 
monodisperse particles at $\mathrm{t}=\infty, \mathrm{n}_{+}^{\infty}$, is given from the following equation ${ }^{29}$

$$
\mathrm{n}_{+}^{\infty}=\frac{Q_{0} V_{m}}{v_{+}^{*}}
$$

where $Q_{0}$ is the supply rate of the solute, $V_{m}$ is the mole volume of the solute, and $v_{+}^{*}$ is the mean volume growth rate of the stable nuclei. In our case, $Q_{0}$ is determined by the mixing rate of antisolvent, as adding anti-solvent resulted in decreased solubility of zein and therefore super-saturation of zein. The degree of super-saturation is positively correlated to the nucleation rate of the solute and therefore determines the number of stable nuclei. ${ }^{20}$ Thus, the high mixing rate means a high degree of super-saturation would occur and result in more nuclei. Burst nucleation will consume a large amount of supersaturated zein, and ultimately the zein concentration would reach the critical nucleation concentration below which nucleation cannot occur and allow for nuclei to begin to grow. Although nucleus aggregation may be one of the routes of particle formation, the original number of nuclei will determine the final particle number. As a result, rapid mixing results in a large particle number and therefore smaller particle size. The correlation of mixing rate and particle size was demonstrated by the data shown in Figure 3. In further support of the previously mentioned speculation, we determined the photon count rate of each sample using dynamic light scattering technology with the constant measurement position and attenuation factor, and then the ratios of particle numbers of all samples were calculated using the following equation ${ }^{37}$

$$
\frac{P_{1}}{P_{2}}=\frac{C_{1} d_{1}^{6}}{C_{2} d_{2}^{6}}
$$

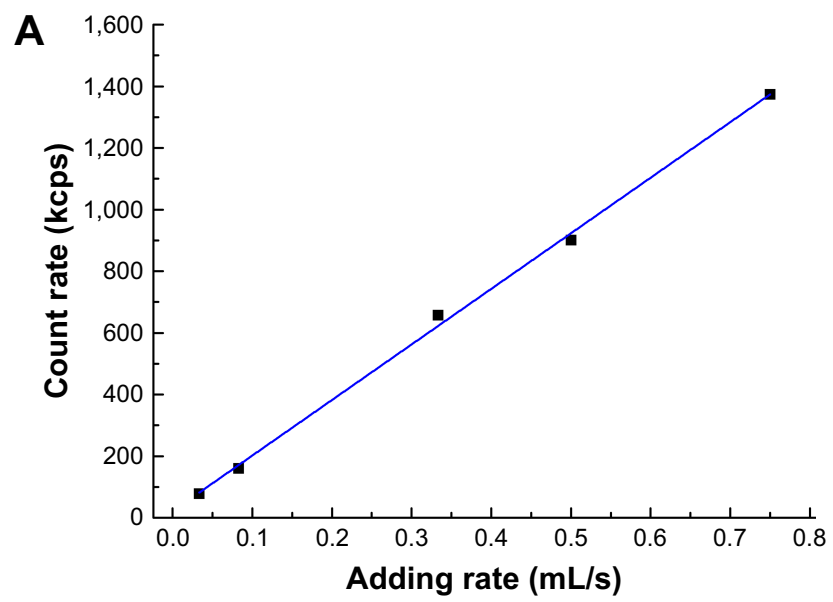

Figure 4 (A) Relationship of mixing rates to count rate of the resultant particles. (B) Relationship of mixing rates to concentrations of the resultant particles (number of particles per $\mathrm{mL})$. where $P_{1}$ and $P_{2}$ are the photon count rates of sample 1 and sample 2, respectively, $C_{1}$ and $C_{2}$ are the number concentrations, and $d_{1}$ and $d_{2}$ are their diameters of the previously mentioned two samples. The relationships between mixing rates and count rates and concentration of the resultant particles (number of particles per $\mathrm{mL}$ ) are shown in Figure 4A and $\mathrm{B}$, respectively.

\section{Zein concentration}

The possible effect of zein concentration on the properties of zein nanoparticles was also investigated. In this experiment, the zein concentration varied from $4 \mathrm{mg} / \mathrm{mL}$ to $19 \mathrm{mg} / \mathrm{mL}$ in aqueous ethanol solution, and the mixing rate of $30 \mathrm{~mL} / \mathrm{min}$ was used as a representative mixing rate. High zein concentrations can lead to large aggregates in starting point, while low concentrations may affect the homogenicity of the resultant zein particles. The results are shown in Table 2. Expectedly, increasing the zein concentration resulted in an increased particle size and higher PDI values. Approximately a 5-fold higher zein concentration led to a limited increase in particle size from $170 \mathrm{~nm}$ to $275 \mathrm{~nm}$. These results indicated that it is difficult to obtain zein nanoparticles larger than $300 \mathrm{~nm}$ with even size distribution by only changing the zein concentration under the current experiment conditions. The limited increase in size may be due to the increased number of nuclei resulting from the high zein concentrations. This speculation is also supported by the previously mentioned fundamental equation for size control of monodisperse systems. ${ }^{29}$ Based on our finding of slow mixing rate contributing to large particle size, we attempted to combine the zein concentration factor with mixing rate to extend the size scale into the micro range. The results are shown in Table 2. Ternary phase compositions corresponding to formulations

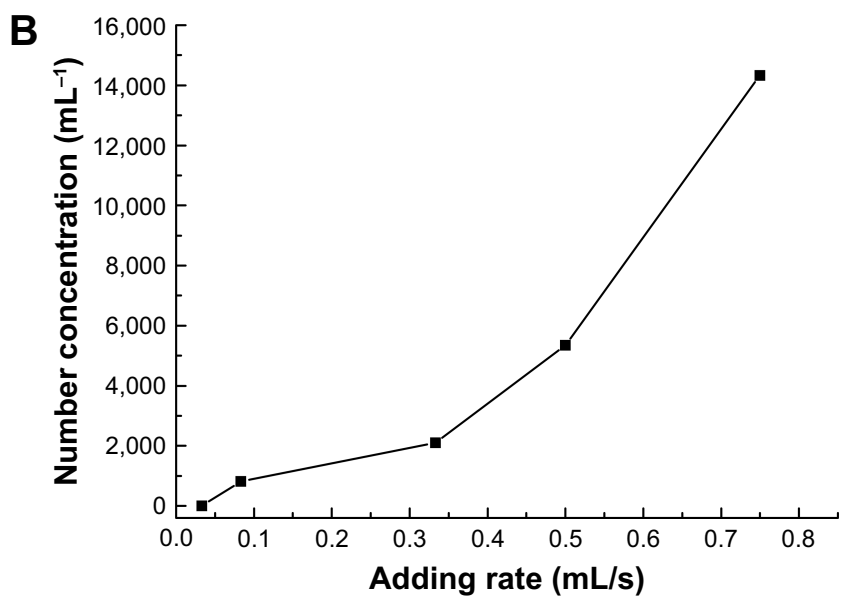


Table 2 Characterization of particles fabricated at different zein concentrations and mixing rates

\begin{tabular}{llllll}
\hline Formulation & Zein $\mathbf{( g )}$ & $\begin{array}{l}\text { Mixing rate } \\
(\mathbf{m L} / \mathbf{m i n})\end{array}$ & $\begin{array}{l}\text { Z-average } \\
\text { size }(\mathbf{n m})\end{array}$ & PDI & $\begin{array}{l}\text { Zeta potential } \\
(\mathbf{m V})\end{array}$ \\
\hline 1 & 0.02 & 30 & $168.6 \pm 1.60$ & $0.044 \pm 0.011$ & $25.5 \pm 2.71$ \\
2 & 0.025 & 30 & $169.2 \pm 0.70$ & $0.076 \pm 0.012$ & $37.1 \pm 2.97$ \\
3 & 0.035 & 30 & $192.0 \pm 2.36$ & $0.067 \pm 0.026$ & $37.2 \pm 0.55$ \\
4 & 0.05 & 30 & $197.4 \pm 3.73$ & $0.032 \pm 0.027$ & $40.2 \pm 0.26$ \\
5 & 0.065 & 30 & $209.6 \pm 1.08$ & $0.181 \pm 0.007$ & $45.8 \pm 0.44$ \\
6 & 0.08 & 30 & $241.2 \pm 2.48$ & $0.206 \pm 0.003$ & $45.6 \pm 1.14$ \\
7 & 0.095 & 30 & $275.3 \pm 2.94$ & $0.217 \pm 0.014$ & $46.7 \pm 0.53$ \\
8 & 0.1 & 6 & $472.9 \pm 8.30$ & $0.089 \pm 0.018$ & $43.2 \pm 0.81$ \\
9 & 0.1 & 3.87 & $656.0 \pm 1.65$ & $0.255 \pm 0.012$ & $44.4 \pm 1.78$ \\
10 & 0.2 & 3.87 & $1,271.7 \pm 30.17$ & $0.193 \pm 0.022$ & $46.9 \pm 1.63$ \\
11 & 0.3 & 6 & $1,159.3 \pm 95.55$ & $0.269 \pm 0.158$ & $49.5 \pm 0.81$ \\
\hline
\end{tabular}

Note: Data presented as mean \pm standard deviation.

Abbreviation: PDI, polydispersity index.

1 to 11 after mixing are shown in Figure 5. As expected, with increasing zein concentrations and decreasing mixing rates, larger particle sizes were achieved accompanied with broader size distributions. However, in all cases, PDI values were less than 0.3 , suggesting an acceptable level of polydispersity. The previously mentioned results suggested that selecting the appropriate combination of zein concentration with mixing rate is a feasible strategy to obtaining the desired zein particles with different sizes and low PDI values.

\section{Insight into formation of zein nanoparticles}

Timescale of particle formation

To illustrate the possible effect of ethanol evaporation on particle properties, we examined the particle size and PDI of the resultant zein nanoparticles at different time intervals during

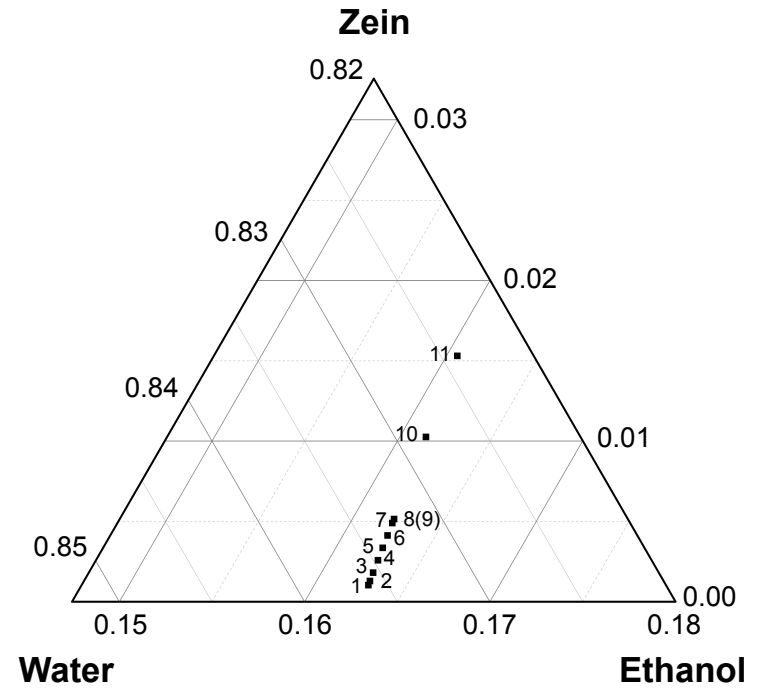

Figure 5 Ternary phase compositions of formulation I to II after mixing $80 \%$ ethanol solution containing zein with deionized water. a $3 \mathrm{~h}$ evaporation process. In this experiment, the mixing rate of $30 \mathrm{~mL} / \mathrm{min}$ was used. The results are shown in Figure 6. Unexpectedly, no remarkable change occurred in either size or PDI during the ethanol evaporation, suggesting that particle formation and growth occurred during the mixing process. To further illustrate the timescale of nanoparticle formation, we performed a video framing assay of Tyndall scattering recorded in a darkroom during the addition of zein-containing solvent to the anti-solvent phase with or without stirring. ${ }^{38}$ Results showed that zein nanoprecipation occurred in $0.36 \mathrm{~s}$ under the static condition, while an obvious Tyndall phenomenon was observed in $0.16 \mathrm{~s}$ under the stirring condition. In considering the interference resulting from the interface vibration, the onset of precipitation may occur in a shorter timescale. This reasoning may explain why the particle size did not show any change at all sampling time points. Interestingly, zeta potential values of particles increased with time and

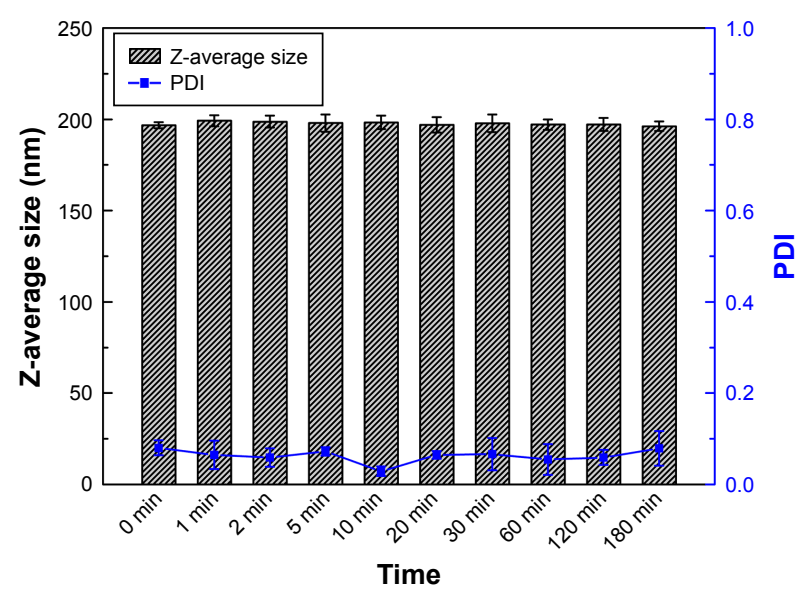

Figure 6 Characterization of resultant zein nanoparticles at different time intervals during $3 \mathrm{~h}$ evaporation process.

Abbreviation: PDI, polydispersity index. 
finally reached a plateau. This increase of zeta potentials likely resulted from the gradual absorption of charged ions onto the particle surface during ethanol evaporation. The charged ions may have originated from the zein powder as both ethanol and deionized water are free of ions. The contribution of different ionization states of zein in co-solvent systems to zeta potential is excluded since the zeta-potential assay was performed in an extensively diluted solution with $1 \mathrm{mM}$ sodium chloride. The previously mentioned results revealed that particle formation in this study may be different from the cases reported before, in which ethanol evaporation induced layer-by-layer adsorption of zein to a central core or nucleus. ${ }^{39,40}$ The layer-by-layer assembly requires a long time and slow ethanol evaporation without stirring, and thus burst nucleation and uniform particle growth would be less likely to occur.

\section{Temperature effect}

According to the classic nucleation theory, both solute and nucleus diffusion have a significant influence on the process of nucleus growth during particle formation and therefore may influence the final particle size. A low temperature usually leads to more limited diffusion and therefore less nucleus aggregation compared with that at a high temperature. As a result, more nuclei would experience uniform growth which in turn decreases the final particle size. However, decreasing the temperature will cause a higher degree of super-saturation and more nuclei when the same amount of solute is dispersed into the medium with constant volume. Thus, a small particle size can be expected at low temperatures. To confirm the previously mentioned speculation, zein nanoparticles were prepared at various mixing rates at $8^{\circ} \mathrm{C}-37^{\circ} \mathrm{C}$, respectively, and their physicochemical properties were compared. The related results are shown in Figure 7. As expected, at all

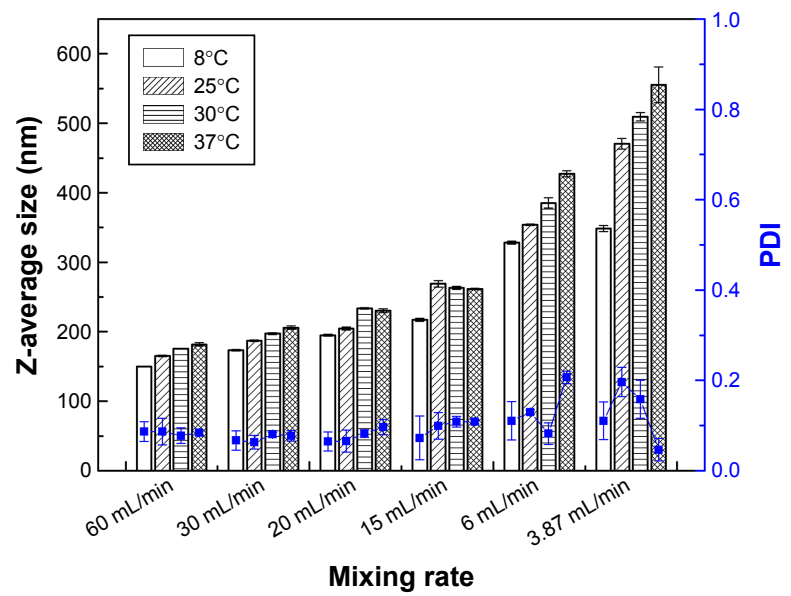

Figure 7 Effect of temperature on the particle size and size distribution of zein nanoparticles.

Abbreviation: PDI, polydispersity index. mixing rates, decreasing the temperature led to smaller particle sizes. However, with the increase in mixing rate, the effect of temperature on particle size could be partly offset, as evidenced by the decreasing gap in sizes between particles fabricated at different temperatures. For example, at the high mixing rate of $60 \mathrm{~mL} / \mathrm{min}$, particle sizes ranged between $149.9 \mathrm{~nm}$ and $181.9 \mathrm{~nm}$. Besides the different particle sizes, no remarkable difference was observed in zeta potential and PDI values in all groups except for the $6 \mathrm{~mL} / \mathrm{min}$ and $3.87 \mathrm{~mL} / \mathrm{min}$ groups. The previously mentioned results indicate that diffusion may be an optional parameter that can be used to control particle size.

\section{Controlled drug release of zein/SC hybrid nanoparticles versus SC-coated zein nanoparticles}

In order to improve the colloidal stability of zein nanoparticles, SC was introduced into the previously mentioned system. A similar strategy was first reported by Patel et al. ${ }^{23}$ The mechanism of improved stability and redispersibility of zein particles is SC-mediated electrostatic repulsion. In this study, considering the high solubility and dispersity of SC in water, it was introduced into water to form a solution before mixing. Unfortunately, adding $15 \mathrm{~mL}$ SC solution into $5 \mathrm{~mL}$ aqueous ethanol solution containing zein led to large aggregates due to the limited SC solubility in the mixed system; meanwhile, mixing these solutions in the inverse process resulted in even and fine zein/SC hybrid nanoparticles. Further investigation revealed that the resultant hybrid nanoparticles displayed size of approximately $120 \mathrm{~nm}$ that was independent of the mixing rate. However, a negative zeta potential of -23 to $-27 \mathrm{mV}$ was observed, suggesting the presence of SC on the outer surface of particles. The mechanism of particle formation may involve two processes. One is the SC adsorption-mediated growth arrest of zein nuclei or nanoparticles, and another is zein/ SC coacervation-mediated nanoprecipitation during mixing. As both could occur in a short time under stirring condition, the resultant zein/SC nanoparticles are probably hybrid. An attempt to obtain size-controlled and structuredefined zein/SC nanoparticles was made by directly adding the preformed size-controlled zein nanoparticles into the $\mathrm{SC}$ solution. As zein nanoparticles were positively charged and formed before mixing with SC-contained solution, the electrostatic interaction of negatively charged SC with zein can only occur on the surface of the already formed zein nanoparticles. As a result, zein/SC nanoparticles with a core-shell structure may be obtained. As expected, slightly increased 
particle size and reversed zeta potential of -28 to $-30 \mathrm{mV}$ were found with the resultant nanoparticles, indicating the absorption of SC onto the surface of the zein nanoparticles. The previously mentioned expectation was further confirmed by the different zeta potential values of zein/SC hybrid nanoparticles and SC-coated zein nanoparticles prepared in series of zein/SC weight ratios from 25:1 to 1:1 (data not shown). In the same conditions, SC-coated zein nanoparticles always showed lower zeta potential values than those of zein/SC hybrid nanoparticles, indicating less distribution of SC on the outer surface of zein/SC hybrid nanoparticles. It should be noted that, for both cases, no chemical interactions between zein and SC happened during particle formation as evidenced by the FTIR spectra (Figure 8). However, compared with the spectrum of zein/SC physical mixture, peak shifts from $1,664.5$ to $1,660.6 \mathrm{~cm}^{-1}$ for zein/SC hybrid nanoparticles and $1,664.5$ to $1,658.7 \mathrm{~cm}^{-1}$ for SC-coated zein nanoparticles were observed, which may be due to increasing $\mathrm{NH}-\mathrm{O}$ hydrogen bond interactions. ${ }^{23}$ Further investigation is needed to illustrate the possible conformation changes of zein and SC during particle formation. Based on the previously mentioned data, it is reasonable to conclude that the core-shell structure of SC-coated zein nanoparticles was very likely different from that of zein/SC hybrid nanoparticles prepared by the previously mentioned inverse mixing method.

Thus, the drug loading efficiency and release profiles were investigated and compared in the subsequent experiments. Here, coumarin 6 was used as a hydrophobic model drug. Results of the investigation of drug loading efficiency are shown in Figure 9A, and drug release profiles of coumarin 6 are presented in Figure 9B. A slightly higher encapsulation efficiency was achieved for core-shell SCcoated zein nanoparticles than those of zein/SC hybrid nanoparticles. Due to the intrinsic hydrophobicity of coumarin 6 , both fabrication methods resulted in a high encapsulation efficiency of over $90 \%$. In the case of drug release, all zein/ $\mathrm{SC}$ hybrid nanoparticles presented a burst release phase in the first $0.5 \mathrm{~h}$, which was followed by a slow release during the rest of the time. In addition, at all sampling time points, no significant difference was observed between the three formulations. The similar release profiles may be due to the similar particle sizes of approximately $150 \mathrm{~nm}$, indicating the limited capacity of zein/SC hybrid nanoparticles in controlled drug release. In contrast, core-shell SC-coated zein nanoparticles showed mixing rate-dependent drug release profiles in both the burst release phase and the subsequent delayed release phase (Figure 9C). The core-shell nanoparticles fabricated using a low mixing rate displayed alleviated burst release and decreased accumulative release when compared with those fabricated under high mixing rates. The correlation can be explained by the different particle sizes resulting from the different mixing rates. As presented in the results section on the effect of mixing rate, the high mixing rate usually led to a small particle size and

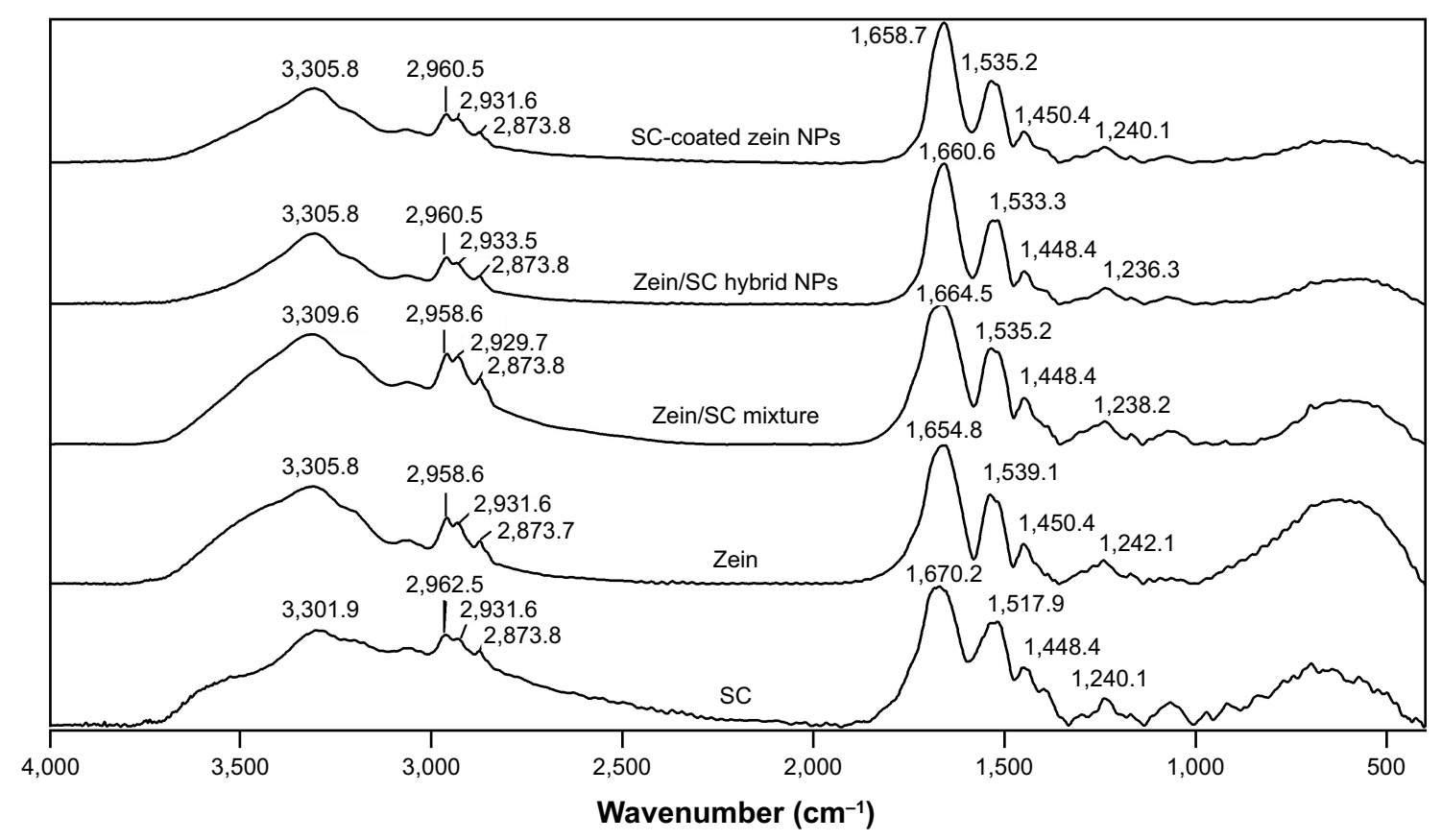

Figure 8 FTIR spectra of SC, zein, zein/SC mixture with I:I weight ratio, zein/SC hybrid nanoparticles, and SC-coated zein nanoparticles. Abbreviations: FTIR, Fourier transform infrared; NPs, nanoparticles; SC, sodium caseinate. 


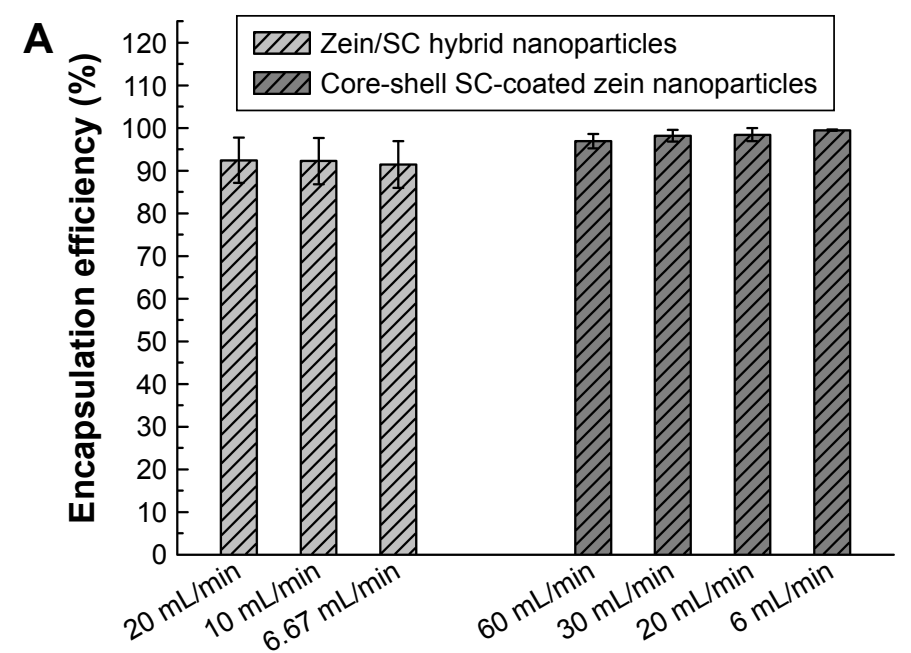

\section{Adding rate}
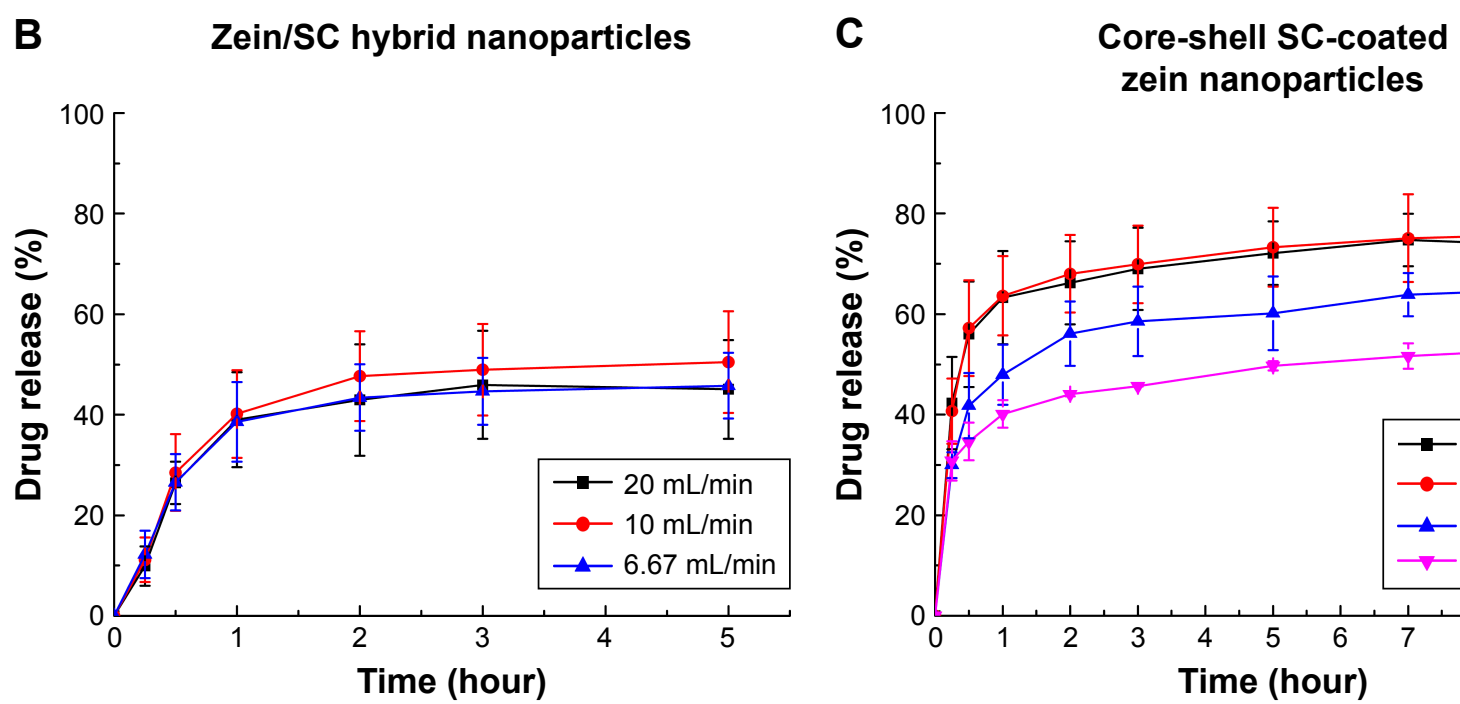

Figure 9 (A) Encapsulation efficiency of coumarin 6-loaded zein/SC hybrid nanoparticles and core-shell SC-coated zein nanoparticles; (B) release profiles of zein/SC hybrid nanoparticles fabricated at the mixing rate of 20,10 , and $6.67 \mathrm{~mL} / \mathrm{min}$. (C) Release profiles of core-shell SC-coated zein nanoparticles fabricated at the mixing rates of $60,30,20$, and $6 \mathrm{~mL} / \mathrm{min}$.

Abbreviation: SC, sodium caseinate.

therefore large surface area of particles. According to the Noyes-Whitney equation, a large surface area in contact with the release medium can improve the solute dissolution rate and therefore cause rapid release ${ }^{41}$ It is worth noting that the formulations in Figure 9C have the same composition but were only subject to different mixing rates during fabrication. These results suggest that controlled drug release can be achieved through obtaining different particle sizes by fine-tuning the mixing rate.

\section{Conclusion}

In this study, size-controlled zein nanoparticles with low polydispersity were prepared by a modified phase separation method without the requirement of special devices, such as well-designed microfluidic devices or chamber mixers. Moreover, the size of zein particles could be easily extended from nanometer to micrometer scale without a significant influence on polydispersity by adjusting the two key factors of mixing rate and zein concentration. Particle formation followed the classical nucleation/growth theory, and diffusion could exert a significant influence on the process of particle formation and growth. In addition, size-controlled SC-coated zein nanoparticles could also be easily prepared using preformed zein nanoparticles as cores. The resultant particles with the core-shell structure were demonstrated to have greater advantages than hybrid zein/SC nanoparticles in both encapsulation efficiency and drug release of hydrophobic drugs. 


\section{Acknowledgments}

This work was supported by the Fundamental Research Funds for the Central Universities (grant no 101832013) and the Jilin Province Science and Technology Development Plan (grant no 20140309007YY). We thank the anonymous reviewers and the editor for their constructive comments and suggestions.

\section{Disclosure}

The authors report no conflicts of interest in this work.

\section{References}

1. Maham A, Tang Z, Wu H, Wang J, Lin Y. Protein-based nanomedicine platforms for drug delivery. Small. 2009;5(15):1706-1721.

2. Corradini E, Curti PS, Meniqueti AB, Martins AF, Rubira AF, Muniz EC. Recent advances in food-packing, pharmaceutical and biomedical applications of zein and zein-based materials. Int J Mol Sci. 2014;15(12): 22438-22470.

3. Zhang Y, Cui L, Che X, et al. Zein-based films and their usage for controlled delivery: Origin, classes and current landscape. J Control Release. 2015;206:206-219.

4. Zhang Y, Cui L, Chen Y, et al. Zein-based nanofibres for drug delivery: classes and current applications. Curr Pharm Des. 2015;21(22): 3199-3207.

5. Zhang Y, Cui L, Li F, et al. Design, fabrication and biomedical applications of zein-based nano/micro-carrier systems. Int J Pharm. 2016; 513(1-2):191-210.

6. Holding DR. Recent advances in the study of prolamin storage protein organization and function. Front Plant Sci. 2014;5:276.

7. Anderson TJ, Lamsal BP. Zein extraction from corn, corn products, and coproducts and modifications for various applications: a review. Cereal Chem. 2011;88(2):159-173.

8. Lee S, Kim YC, Park JH. Zein-alginate based oral drug delivery systems: protection and release of therapeutic proteins. Int J Pharm. 2016; 515(1-2):300-306.

9. Xu H, Shen L, Xu L, Yang Y. Controlled delivery of hollow corn protein nanoparticles via non-toxic crosslinking: in vivo and drug loading study. Biomed Microdevices. 2015;17(1):8.

10. Xu HL, Jiang QR, Reddy N, Yang YQ. Hollow nanoparticles from zein for potential medical applications. J Mater Chem. 2011;21(45): 18227-18235.

11. Podaralla S, Averineni R, Alqahtani M, Perumal O. Synthesis of novel biodegradable methoxy poly(ethylene glycol)-zein micelles for effective delivery of curcumin. Mol Pharm. 2012;9(9):2778-2786.

12. Suzuki T, Sato E, Matsuda Y, Tada H, Unno K, Kato T. Preparation of zein microspheres conjugated with antitumor drugs available for selective cancer chemotherapy and development of a simple colorimetric determination of drugs in microspheres. Chem Pharm Bull (Tokyo). 1989; 37(4):1051-1054.

13. Podaralla $S$, Perumal O. Influence of formulation factors on the preparation of zein nanoparticles. AAPS PharmSciTech. 2012;13(3):919-927.

14. Zhong Q, Jin M. Nanoscalar structures of spray-dried zein microcapsules and in vitro release kinetics of the encapsulated lysozyme as affected by formulations. J Agric Food Chem . 2009;57(9):3886-3894.

15. Chen HQ, Zhong QX. Processes improving the dispersibility of spraydried zein nanoparticles using sodium caseinate. Food Hydrocoll. 2014; 35:358-366.

16. Gomez-Estaca J, Balaguer MP, Gavara R, Hernandez-Munoz P. Formation of zein nanoparticles by electrohydrodynamic atomization: effect of the main processing variables and suitability for encapsulating the food coloring and active ingredient curcumin. Food Hydrocoll. 2012;28(1):82-91.
17. Karthikeyan K, Lakra R, Rajaram R, Korrapati PS. Development and characterization of zein-based micro carrier system for sustained delivery of aceclofenac sodium. AAPS PharmSciTech. 2012;13(1):143-149.

18. Chen L, Hebrard G, Beyssac E, Denis S, Subirade M. In vitro study of the release properties of soy-zein protein microspheres with a dynamic artificial digestive system. J Agric Food Chem. 2010;58(17):9861-9867.

19. Aubry J, Ganachaud F, Cohen Addad JP, Cabane B. Nanoprecipitation of polymethylmethacrylate by solvent shifting: 1. Boundaries. Langmuir. 2009;25(4):1970-1979.

20. Horn D, Rieger J. Organic nanoparticles in the aqueous phasetheory, experiment, and use. Angew Chem Int Ed Engl. 2001;40(23): 4330-4361.

21. Zhong Q, Jin M. Zein nanoparticles produced by liquid-liquid dispersion. Food Hydrocol. 2009;23(8):2380-2387.

22. Patel AR, Hu Y, Tiwari JK, Velikov KP. Synthesis and characterisation of zein-curcumin colloidal particles. Soft Matter. 2010;(6):6192-6199.

23. Patel AR, Bouwens EC, Velikov KP. Sodium caseinate stabilized zein colloidal particles. J Agric Food Chem. 2010;58(23):12497-12503.

24. Zhang Y, Niu Y, Luo Y, Ge M, Yang T, Yu LL, Wang Q. Fabrication, characterization and antimicrobial activities of thymol-loaded zein nanoparticles stabilized by sodium caseinate-chitosan hydrochloride double layers. Food Chem. 2014;142:269-275.

25. Muthuselvi L, Dhathathreyan A. Simple coacervates of zein to encapsulate Gitoxin. Colloids Surf B Biointerfaces. 2006;51(1):39-43.

26. Luo Y, Teng Z, Wang TT, Wang Q. Cellular uptake and transport of zein nanoparticles: effects of sodium caseinate. J Agric Food Chem. 2013; 61(31):7621-7629.

27. Choi JS, Cao J, Naeem M, Noh J, Hasan N, Choi HK, Yoo JW. Sizecontrolled biodegradable nanoparticles: preparation and size-dependent cellular uptake and tumor cell growth inhibition. Colloids Surf B Biointerfaces. 2014;122:545-551.

28. Ahmed OA, Hosny KM, Al-Sawahli MM, Fahmy UA. Optimization of caseinate-coated simvastatin-zein nanoparticles: improved bioavailability and modified release characteristics. Drug Des Devel Ther. 2015;9:655-662.

29. Sugimoto T. Monodispersed Particles. Amsterdam: Elsevier; 2001.

30. Song J, Kim D, Lee D. Size control in the synthesis of 1-6 nm gold nanoparticles via solvent-controlled nucleation. Langmuir. 2011;27(22): 13854-13860.

31. Mossé J. Monographie sur une protéine du mals: la zéine [Monograph on a maize protein: zein]. Ann Physiol. 1961;3:105-139. French.

32. Comert F, Malanowski AJ, Azarikia F, Dubin PL. Coacervation and precipitation in polysaccharide-protein systems. Soft Matter. 2016; 12(18):4154-4161.

33. Mishra M. Handbook of Encapsulation and Controlled Release. Boca Raton, Florida: CRC Press Taylor \& Francis Group; 2016.

34. Hickey JW, Santos JL, Williford JM, Mao HQ. Control of polymeric nanoparticle size to improve therapeutic delivery. $J$ Control Release. 2015;219:536-547.

35. Capretto L, Carugo D, Mazzitelli S, Nastruzzi C, Zhang X. Microfluidic and lab-on-a-chip preparation routes for organic nanoparticles and vesicular systems for nanomedicine applications. Adv Drug Deliv Rev. 2013;65(11-12):1496-1532.

36. Xie H, Smith JW. Fabrication of PLGA nanoparticles with a fluidic nanoprecipitation system. J Nanobiotechnology. 2010;8:18.

37. Shang J, Gao X. Nanoparticle counting: towards accurate determination of the molar concentration. Chem Soc Rev. 2014;43(21):7267-7278.

38. Jindal AB, Devarajan PV. Asymmetric lipid-polymer particles (LIPOMER) by modified nanoprecipitation: role of non-solvent composition. Int J Pharm. 2015;489(1-2):246-251.

39. Wang Y, Padua GW. Formation of zein microphases in ethanol-water. Langmuir. 2010;26(15):12897-12901.

40. Wang Y, Padua GW. Nanoscale characterization of zein self-assembly. Langmuir. 2012;28(5):2429-2435.

41. Dokoumetzidis A, Macheras P. A century of dissolution research: from Noyes and Whitney to the biopharmaceutics classification system. Int J Pharm. 2006;321(1-2):1-11. 
International Journal of Nanomedicine

Dovepress

\section{Publish your work in this journal}

The International Journal of Nanomedicine is an international, peerreviewed journal focusing on the application of nanotechnology in diagnostics, therapeutics, and drug delivery systems throughout the biomedical field. This journal is indexed on PubMed Central, MedLine, CAS, SciSearch ${ }^{\circledR}$, Current Contents ${ }^{\circledR} /$ Clinical Medicine,
Journal Citation Reports/Science Edition, EMBase, Scopus and the Elsevier Bibliographic databases. The manuscript management system is completely online and includes a very quick and fair peer-review system, which is all easy to use. Visit http://www.dovepress.com/ testimonials.php to read real quotes from published authors.

Submit your manuscript here: http://www.dovepress.com/international-journal-of-nanomedicine-journal 